\title{
Combinatorics, Probability and Computing
}

http://journals.cambridge.org/CPC

Additional services for Combinatorics, Probability and Computing:

Email alerts: $\underline{\text { Click here }}$

Subscriptions: $\underline{\text { Click here }}$

Commercial reprints: $\underline{\text { Click here }}$

Terms of use : $\underline{\text { Click here }}$

\section{Point Selections and Weak $\varepsilon$-Nets for Convex Hulls}

Noga Alon, Imre Bárány, Zoltán Füredi and Daniel J. Kleitman

Combinatorics, Probability and Computing / Volume 1 / Issue 03 / September 1992, pp 189 - 200

DOI: 10.1017/S0963548300000225, Published online: 12 September 2008

Link to this article: http://journals.cambridge.org/abstract S0963548300000225

How to cite this article:

Noga Alon, Imre Bárány, Zoltán Füredi and Daniel J. Kleitman (1992). Point Selections and Weak $\varepsilon$-Nets for Convex Hulls. Combinatorics, Probability and Computing, 1, pp 189-200 doi:10.1017/S0963548300000225

Request Permissions : $\underline{\text { Click here }}$ 


\title{
Point Selections and Weak $\varepsilon$-Nets for Convex Hulls
}

\author{
NOGA ALON', IMRE BÁRÁNY ${ }^{\ddagger}$, ZOLTÁN FÜREDI* and \\ DANIEL J. KLEITMAN $§$ \\ † Department of Mathematics, Raymond and Beverly Sackler Faculty of Exact Sciences,
}

Tel Aviv University, Tel Aviv, Israel, and Bellcore, Morristown, NJ 07960, USA.

e-mail: noga@math.tau.ac.il

$\ddagger$ Cowles Foundation, Yale University, New Haven, CT 06520, USA, and Courant Institute, New York University, New York, NY 10009, USA.

e-mail: h2923bar@ella.hu

* Mathematical Institute of the Hungarian Academy of Sciences

POB 127, 1364 Budapest, Hungary,

and Department of Mathematics, University of Illinois, Urbana, IL 61801, USA.

e-mail: zoltan@math.uiuc.edu

$\S$ Department of Mathematics, MIT, Cambridge, MA 02139, USA.

e-mail: djk@math.mit.edu

Received 30 October 1991 ; revised 7 September 1992

One of our results: let $X$ be a finite set on the plane, $0<\varepsilon<1$, then there exists a set $F$ (a weak $\varepsilon$-net) of size at most $7 / \varepsilon^{2}$ such that every convex set containing at least $\varepsilon|X|$ elements of $X$ intersects $F$. Note that the size of $F$ is independent of the size of $X$.

\section{Introduction}

This paper is about weak $\varepsilon$-nets, point selections, convex hulls, and related topics. To explain what they mean, we start with the assumption that $d \geq 2$ and

$$
X \subset \mathbb{R}^{d} \text { is a set of } n \text { points in general position. }
$$

\footnotetext{
† Research supported by a United States Israel BSF grant.

$\mp$ On leave from the Mathematical Institute of the Hungarian Academy of Sciences, POB 127, 1364 Budapest, Hungary. Supported by the Program in Discrete Mathematics and its Applications at Yale, NSF grant 8901484, and Hungarian National Science Foundation grant No. 1812.

* This research was done while the author visited the Department of Mathematics at Massachusetts Institute of Technology. Supported in part by Hungarian National Science Foundation grant No. 1812.
}

$\S$ Supported in part by NSF grants DMS-86-06225 and AFOSR-0271. 
We only assume general position to simplify the presentation: all of our results can be extended to any finite set $X$ using an appropriate limit procedure (and suitable extensions of the definitions). Write $\left(\begin{array}{c}X \\ d+1\end{array}\right)$ for the set of all $(d+1)$-tuples of $X$. Since the points of $X$ live in $\mathbb{R}^{d}$, these $(d+1)$-tuples can, and will, be called simplices when we consider their convex hull. This should not cause any confusion.

We deal with the following three problems. Given a (large) set of simplices $\mathscr{H} \subset\left(\begin{array}{c}X \\ d+1\end{array}\right)$, find a point that is contained in the maximum possible number of simplices. We call this the point selection problem (Section 2, proof in Section 6). In the hitting set problem (Sections 5 and 7), we shall look for a small set $E$ meeting "almost all" simplices in $\left(\begin{array}{c}X \\ d+1\end{array}\right)$. Finally, in the weak $\varepsilon$-net problem (Sections 4 and 8-10), given a set $X$ and $0<\varepsilon<1$, we look for a small set $F$ such that any convex region $C$ with $|X \cap C| \geq \varepsilon|X|$ contains a point of $F$. We shall find several upper bounds for $\min |F|$, together with polynomial algorithms for finding a small set $F$.

\section{Piercing many simplices by one point}

A family $\mathscr{H}$ is called pierceable if there exists a point common to int $\operatorname{conv}(S)$ for every $S \in \mathscr{H}$. We have the following Point Selection Theorem.

Theorem 2.1. Given $d \geq 2$, there exists a constant $s=s_{d}$ such that any family $\mathscr{H} \subset\left(\begin{array}{c}X \\ d+1\end{array}\right)$, with $|\mathscr{H}|=p\left(\begin{array}{c}n \\ d+1\end{array}\right)$, contains a pierceable subfamily $\mathscr{H}^{\prime}$ such that

$$
\left|\mathscr{H}^{\prime}\right| \gg_{d} p^{s}\left(\begin{array}{c}
n \\
d+1
\end{array}\right)
$$

Here, and in what follows, we are using Vinogradov's notation. For two functions $f$ and $g, f \gg g$ means that there are two absolute constants $c_{1}>0$ and $c_{2} \in \mathbb{R}$ such that $f \geq c_{1} g+c_{2}$ for all values of the parameters. Similarly, $f \gg_{d} g$ means that there are constants $c_{1}(d)>0$ and $c_{2}(d)$ such that $f \geq c_{1}(d) g+c_{2}(d)$ for all values of the parameters.

The first point selection theorem is due to Boros and Füredi [7]. They show that for $d=2$, the family $\left(\begin{array}{l}X \\ 3\end{array}\right)$ contains a pierceable subfamily of size $(2 / 9)\left(\begin{array}{l}n \\ 3\end{array}\right)$. This is extended for any dimension in Bárány [3], where it is proved that $\left(\begin{array}{c}X \\ d+1\end{array}\right)$ contains a pierceable subfamily of size at least

$$
(d+1)\left(\begin{array}{c}
\lfloor n /(d+1)\rfloor \\
d+1
\end{array}\right)=(1-o(1)) \frac{1}{(d+1)^{d}}\left(\begin{array}{c}
n \\
d+1
\end{array}\right) .
$$

The term "point selection" comes from Aronov et al. [2]. They prove, again when $d=2$, that any family $\mathscr{H} \subset\left(\begin{array}{l}X \\ 3\end{array}\right)$ of size $n^{3-\alpha}$ contains a pierceable subfamily of size

$$
2^{-9} \frac{n^{3-3 \alpha}}{\log ^{5} n}
$$

Thus, $s=s_{2}=3+\delta$ will do (for any positive $\delta$ ) in the point selection theorem in the range $p=n^{-\alpha}, \alpha>0$. Here we prove that, in general, one can take

$$
s_{d}=(4 d+1)^{d+1} .
$$




\section{The multicoloured Tverberg theorem}

Our point selection theorem will follow from a nice recent result of Živaljević and Vrećica [19], which was also conjectured in [4]. The result is a "multicoloured" version of Tverberg's theorem [16]. One form of the latter says that any set of $(d+1) t$ points in $\mathbb{R}^{d}$ can be partitioned into $t$ sets, $S_{1}, \ldots, S_{t}$, each of cardinality $d+1$, so that

$$
\bigcap_{i=1}^{t} \operatorname{conv}\left(S_{i}\right) \neq \emptyset \text {. }
$$

In the multicoloured version, the $(d+1) t$ points come in $d+1$ classes $C_{1}, \ldots, C_{d+1}$, or colours, each of cardinality $t$, and one wants to find "many" pairwise disjoint sets $S_{1}, \ldots$, $S_{r}$, each of cardinality $d+1$, such that every $S_{i}$ is multicoloured (i.e., $\left|S_{i} \cap C_{j}\right|=1$ for every $i$ and $j$ ) and

$$
\bigcap_{i=1}^{r} \operatorname{conv}\left(S_{i}\right) \neq \emptyset .
$$

The question is how large $t=T(r, d)$ must be in order to ensure the existence of such sets $S_{1}, \ldots, S_{r}$. In the planar case, one can take $T(r, 2)=r$ (see [5] or [12]) and this is clearly best possible. Using tools of algebraic topology, Živaljević and Vrecica [19] show that

$$
T(r, d) \leq 2 p(r)-1,
$$

where $p(r)$ is the smallest prime that is not smaller than $r$. It is well known that $p(r) \leq 2 r-1$, whence $T(r, d) \leq 4 r-3$. We will see later that this is where the value in (4) comes from.

\section{Piercing all large convex sets}

A set $F \subset \mathbb{R}^{d}$ is called a weak $\varepsilon$-net for $X$ if, for every $Y \subset X$ with $|Y| \geq \varepsilon n$, the intersection $F \cap \operatorname{conv}(Y)$ is nonempty. At a DIMACS workshop in 1990, E. Welzl [17] asked whether there exists a weak $\varepsilon$-net for $X$ whose size depends only on $\varepsilon$ and $d$. This had been proved true in the planar case in [4] before Welzl posed his question; however, the bound $O\left(\varepsilon^{-1026}\right)$ given in [4] is enormous compared to the bound in the following weak $\varepsilon$-net theorem.

Theorem 4.1. For any $X \subset \mathbb{R}^{d}$ there exists a weak $\varepsilon$-net $F$ with

$$
|F| \ll_{d} \varepsilon^{-(d+1)(1-1 / s)} .
$$

Here, $s$ is the constant $s_{d}$ of the point selection theorem. In the planar case, (3) gives $s_{2}=3+\delta$, i.e., a weak $\varepsilon$-net of size $O\left(\varepsilon^{-\left(2+\delta^{\prime}\right)}\right)$ for any positive $\delta^{\prime}$. We present here a separate argument for the planar case.

Theorem 4.2. For any $X \subset \mathbb{R}^{2}$ there exists a weak $\varepsilon$-net of size $7 \varepsilon^{-2}$.

The proof works in any dimension but gives $O\left(\varepsilon^{-2^{d-1}}\right)$ for the size of the weak $\varepsilon$-net; for $d>2$, this bound is worse than the bound in Theorem 4.1. Also, in Section 10 we 
give an algorithm with running time $O(n \log (1 / \varepsilon))$, which, for a planar set, yields an $\varepsilon$-net of size $O\left(\varepsilon^{-4.818 \cdots}\right)$.

Define

$$
f_{d}(\varepsilon)=\max \min \{|F|: F \text { is a weak } \varepsilon \text {-net for } X\},
$$

where the maximum is taken over all $X$ satisfying (1). It is clear that $f_{d}(\varepsilon) \geq 1 / \varepsilon$, so

$$
\frac{1}{\varepsilon} \leq f_{d}(\varepsilon) \ll_{d} \varepsilon^{-(d+1)\left(1-1 / s_{d}\right)} .
$$

It is not known whether $\varepsilon f_{d}(\varepsilon)$ is bounded when $\varepsilon$ tends to 0 .

Weak $\varepsilon$-nets and the discrepancy of triangles. Consider the case when $X$ is a set of $n$ points chosen randomly, independently, and uniformly from the unit square. When $\varepsilon$ is fixed and $n$ is large, every triangle of area $\varepsilon$ (and contained in the unit square) will contain about $\varepsilon n$ points of $X$. Using this one can show that there is a weak $\varepsilon$-net $F$ for $X$ of size $O((1 / \varepsilon) \log (1 / \varepsilon))$. On the other hand, finding a lower bound for $|F|$ leads to the following old problem of Danzer (see [6] page 285) about irregularities of distributions. How many points are needed to hit every triangle of area $\varepsilon$ contained in the unit square?

When $X$ is the vertex set of a regular $n$-gon in the plane, there is a weak $\varepsilon$-net of size $O\left((1 / \varepsilon) 2^{\log ^{*}(1 / \varepsilon)}\right)$, where $\log ^{*} m$ denotes the function defined by the recursion $\log ^{*}\left(2^{x}\right)=1+\log ^{*} x$ and $\log ^{*} 1=0$. This is a result of Capoyleas [8].

We do not know how large the smallest weak $\varepsilon$-net is for a set of $n$ distinct points on the moment's curve $\left\{\left(t, t^{2}, \ldots, t^{d}\right):-\infty<t<\infty\right\}$ in $\mathbb{R}^{d}$.

A generalization of Helly's theorem. In [1] the above Theorem 4.1 is combined with some additional tools to prove the following Helly-type result, solving an old problem of Hadwiger and Debrunner.

Theorem. [1] For every $p \geq q \geq d+1$, there is a (finite) $c=c(p, q, d+1$ ) such that the following holds: for every family $\mathscr{K}$ of compact convex sets in $\mathbb{R}^{d}$ with the property that among any $p$ members of the family some $q$ are pierceable, there is a set $F$ of at most $c$ points in $\mathbb{R}^{d}$ so that every member of $\mathscr{K}$ contains at least one point of $F$.

An easy consequence of Theorem 4.1 is the following result.

Proposition 4.1. For every $\eta>0$ and for every integer $d$, there is a $c=c(\eta, d)$ such that for every probability measure $\mu$ on $\mathbb{R}^{d}$ there is a set $F$ of at most $c$ points in $\mathbb{R}^{d}$ so that every compact, convex set $C$ of measure $\mu(C) \geq \eta$ contains at least one point of $F$.

Let us sketch a proof of this result. By a usual compactness argument, it is enough to prove the proposition for any finite family $\left\{C_{1}, \ldots, C_{N}\right\}$ of compact convex sets, with $\mu\left(C_{i}\right) \geq \eta$ for every $i$. Choose points $x_{1}, \ldots, x_{n}$ randomly, independently, and according to the distribution $\mu$. Set $X=\left\{x_{1}, \ldots, x_{n}\right\}$. A straightforward application of the large deviation theorem of Chernoff [9] says that the probability that 
$\left|X \cap C_{i}\right| \leq \frac{\eta}{2}|X|$ is very small. This shows that, with positive probability, for large enough $n$ we have

$$
\left|X \cap C_{i}\right| \geq \frac{\eta}{2}|X| \text { for every } i=1, \ldots, N .
$$

Fix such an $X$ and let $F$ be a weak $\varepsilon$-net for $X$, where $\varepsilon=\eta / 2$. Then, clearly, $F$ intersects every $C_{i}$ and is of size $O\left(\eta^{(d+1)(1-1 / s)}\right)$, completing the proof.

Another way of proving the proposition is to use the theorem establishing the HadwigerDebrunner conjecture. Namely, one can show easily that the family of all convex, compact sets whose measure is at least $\eta$ satisfies the conditions of that theorem, with $p=\lceil d / \eta\rceil+1$ and $q=d+1$. This gives that $c(\eta, d) \leq c(p, q, d+1)$. In fact, the first argument given above gives a better bound on $c(\eta, d)$.

\section{Piercing most of the simplices by many points}

It turns out that the point selection theorem is closely related to some other results, which we now describe. We say that a set $E$ misses $S \in\left(\begin{array}{c}X \\ d+1\end{array}\right)$ if $E \cap \operatorname{int} \operatorname{conv}(S)=\emptyset$. (Here, again, $X$ is assumed to satisfy condition (1).) The following hitting set theorem asserts the existence of a "small" set $E$ that misses only "few" members of $\left(\begin{array}{c}X \\ d+1\end{array}\right)$.

Theorem 5.1. For every $\eta>0$ and $X \subset \mathbb{R}^{d}$, there exists a set $E \subset \mathbb{R}^{d}$ that misses at most $\eta\left(\begin{array}{c}n \\ d+1\end{array}\right)$ simplices of $X$ and has size

$$
|E| \ll_{d} \eta^{1-s},
$$

where $s$ is the constant $s_{d}$ in the point selection theorem.

In fact we shall show that the hitting set and the point selection theorems are equivalent.

Observe that $\eta$ may depend on $n=|X|$; for instance, one may take $\eta=n^{-1 / s}$, which gives a set $E$ of size $O\left(n^{1-(1 / s)}\right)$ missing at most $O\left(n^{d+1-1 / s}\right)$ simplices of $X$. This special case of Theorem 4 was proved in [4] for $d=2$ with $s=343$.

We emphasize again that the point selection, the hitting set, and the multicoloured Tverberg theorems are equivalent. In fact, the multicoloured Tverberg theorem with $r=d+1$ implies the point selection theorem with $s=s_{d}=(T(d+1, d))^{d+1}$, and the latter implies the multicoloured Tverberg theorem with $T(r, d) \ll_{d} r$. The equivalence of the point selection and the hitting set theorem is stronger, since it carries over to the exponent $s=s_{d}$. It would be interesting to know the smallest possible exponent $s_{d}$.

Remark on halving planes. As observed in [2] and [4], the point selection theorem (or the hitting set theorem) implies the following upper bound on the number $H_{d}(X)$ of halving hyperplanes a set $X \subset \mathbb{R}^{d}$ can have:

$$
H_{d}(X) \ll_{d} n^{d-1 / s_{d-1}} .
$$

The simplest way of proving this bound is to use the fact that no line meets more than $\left(\begin{array}{c}n \\ d-1\end{array}\right)$ halving simplices. (This was proved in [14] for the planar case, but the argument goes through in $\mathbb{R}^{d}$ without difficulty.) Then the projection of $X$, and of the halving simplices of $X$, to $\mathbb{R}^{d-1}$ gives rise to a family $\mathscr{H}$ in $\mathbb{R}^{d-1}$ (on $n$ points) so that no point 
is contained in more than $\left(\begin{array}{c}n \\ d-1\end{array}\right)$ simplices of $\mathscr{H}$. By the point selection theorem, $\mathscr{H}$ has a pierceable subfamily of size $\gg_{d} p^{s_{d-1}}\left(\begin{array}{l}n \\ d\end{array}\right)$, where $|\mathscr{H}|=p\left(\begin{array}{l}n \\ d\end{array}\right)$. So we get $p \ll_{d} n^{-1 / s_{d-1}}$, as required.

\section{Proof of the point selection theorem}

Here we prove Theorem 2.1. The method is similar to that of [4]. First, we define $V=V(X)$, the set of crossings determined by $d$ distinct hyperplanes through the points of $X$. To this end, let $Q_{1}, \ldots, Q_{d}$ be pairwise disjoint $d$-tuples from $X$. Their crossing is defined as the point of intersection of the hyperplanes $\operatorname{aff}\left(Q_{1}\right), \ldots$, aff $\left(Q_{d}\right)$. Here we assume that $X$ is in a general position, so that any crossing is a well-defined, unique point. To this end, condition (1) can be understood as saying that the coordinates of $X$ are in algebraically independent position. Clearly,

$$
|V|=\frac{1}{d !}\left(\begin{array}{l}
n \\
d
\end{array}\right)\left(\begin{array}{c}
n-d \\
d
\end{array}\right) \ldots\left(\begin{array}{c}
n-d(d-1) \\
d
\end{array}\right),
$$

So that $n^{d^{2}} \ll_{d}|V| \ll_{d} n^{d^{2}}$.

Second, we need a theorem of Erdős and Simonovits [11], which is implicit in Erdős [10] as well.

Theorem. [11] For all positive integers $d$ and $t$, there exists a positive constant $b=b(d, t)$ such that the following holds: if $\mathscr{H}$ is an arbitrary $(d+1)$-graph on $n$ vertices and $p\left(\begin{array}{c}n \\ d+1\end{array}\right)$ edges, where $n^{-t^{-d}} \ll_{d} p \leq 1$, then $\mathscr{H}$ contains at least

$$
b p^{t^{d+1}} n^{(d+1) t}
$$

copies of $K(t, \ldots, t)$, the complete $(d+1)$-partite $(d+1)$-graph with $t$ vertices in each of its $d+1$ vertex classes.

Proof of Theorem 2.1. Consider the family $\mathscr{H} \subset\left(\begin{array}{c}X \\ d+1\end{array}\right)$. Then the Erdoss-Simonovits theorem implies that $\mathscr{H}$ contains at least

$$
b p^{t^{d+1}} n^{(d+1) t}
$$

copies of $K(t, \ldots, t)$, provided $n^{-t^{-d}} \ll_{d} p \leq 1$. Now choose

$$
t=T(d+1, d) \leq 2 p(d+1)-1 \leq 4 d+1
$$

from the multicoloured Tverberg theorem of Živaljević and Vrećica, and consider a copy of $K(t, \ldots, t)$ in $\mathscr{H}$. This consists of $d+1$ pairwise disjoint sets $C_{1}, \ldots, C_{d+1} \subset X$, each of size $t$, such that, for any $x_{1} \in C_{1}, \ldots, x_{d+1} \in C_{d+1}$, the $(d+1)$-tuple $\left\{x_{1}, \ldots, x_{d+1}\right\}$ belongs to $\mathscr{H}$. By the multicoloured Tverberg theorem, there are $d+1$ pairwise disjoint $(d+1)$-tuples $S_{1}, \ldots, S_{d+1}$ such that $\bigcap_{i=1}^{d+1} \operatorname{conv}\left(S_{i}\right)$ is nonempty. The general position of $X$ implies that $\bigcap_{i=1}^{d+1} \operatorname{conv}\left(S_{i}\right)$ is a polytope $P$ with nonempty interior.

The following simple geometric argument shows that there is an $S_{j}$ and there are subsets $Q_{i} \subset S_{i}(i=1, \ldots, d+1, i \neq j)$ with $\left|Q_{i}\right|=d$ such that the crossing of the $Q_{i}$ 's lies in int conv $\left(S_{j}\right)$. Consider a facet $F$ of the polytope $P$. It lies in a (uniquely determined) 
facet of a (uniquely determined) simplex $\operatorname{conv}\left(S_{i}\right)$, say, $\operatorname{conv}\left(S_{1}\right)$. Thus $F$ lies in aff $\left(Q_{1}\right)$ for a (uniquely determined) $Q_{1} \subset S_{1}$, where $\left|Q_{1}\right|=d$. Then the $(d-1)$-dimensional polytope $P_{1}=\operatorname{aff}\left(Q_{1}\right) \cap \bigcap_{i=2}^{d+1} \operatorname{conv}\left(S_{i}\right)$ has nonvoid $(d-1)$-dimensional interior. So it has a facet $F_{1}$ that lies in a facet of one of the $\operatorname{simplices} \operatorname{conv}\left(S_{i}\right)(i \geq 2)$, say $\operatorname{conv}\left(S_{2}\right)$. Thus $F_{1}$ lies in a hyperplane aff $\left(Q_{2}\right)$ for a (uniquely determined) $d$-tuple $Q_{2} \subset S_{2}$. Then the $(d-2)$-dimensional polytope $P_{2}=\operatorname{aff}\left(Q_{1}\right) \cap \operatorname{aff}\left(Q_{2}\right) \cap \bigcap_{i=3}^{d+1} \operatorname{conv}\left(S_{i}\right)$ has nonvoid $(d-2)$ dimensional interior, and so on. We end up with a zero-dimensional polytope, i.e., a singleton

$$
\{v\}=\operatorname{aff}\left(Q_{1}\right) \cap \ldots \cap \operatorname{aff}\left(Q_{d}\right) \cap \operatorname{conv}\left(S_{d+1}\right) .
$$

Then $v$ is a crossing in the interior of $\operatorname{conv}\left(S_{d+1}\right)$.

Now we give a lower bound for the number of pairs $(S, v)$ with $S \in \mathscr{H}, v \in V$, and $v \in$ int $\operatorname{conv}(S)$. Such a pair can be identified with the $(d+1)$-tuple of sets $\left(S, Q_{1}, \ldots, Q_{d}\right)$. As we have seen, every $K(t, \ldots, t)$ contains such a $(d+1)$-tuple with

$$
\bigcap_{i=1}^{d} \operatorname{aff}\left(Q_{i}\right) \subset \text { int } \operatorname{conv}(S) \text {. }
$$

A given $(d+1)$-tuple $\left(S, Q_{1}, \ldots, Q_{d}\right)$ can appear in at most

$$
B n^{t(d+1)-\left(d^{2}+d+1\right)}
$$

copies of $K(t, \ldots, t)$. Consequently

$$
\mid\{(S, v) \in \mathscr{H} \times V: v \in \text { int } \operatorname{conv}(S)\} \mid \geq \frac{\text { number of copies of } K(t, \ldots, t)}{B n^{t(d+1)-\left(d^{2}+d+1\right)}} \gg_{d} p^{d^{d+1}} n^{d^{2}+d+1}
$$

This shows that there is a crossing $v$ in at least

$$
\frac{p^{t^{d+1}} n^{d^{2}+d+1}}{|V|} \gg_{d} p^{t^{d+1}}\left(\begin{array}{c}
n \\
d+1
\end{array}\right)
$$

simplices of $\mathscr{H}$. Let $\mathscr{H}^{\prime}$ be the set of those $(d+1)$-tuples of $\mathscr{H}$ whose convex hull contains $v$. Then, indeed, $\mathscr{H}^{\prime}$ is pierceable and

$$
\left|\mathscr{H}^{\prime}\right| \gg_{d} p^{t^{d+1}}\left(\begin{array}{c}
n \\
d+1
\end{array}\right)
$$

Here $t$ comes from (6). In the hypergraph theorem we needed $p \gg_{d} n^{-t^{-d}}$, but (7) holds trivially if this condition is violated, since then

$$
p^{t^{d+1}}\left(\begin{array}{c}
n \\
d+1
\end{array}\right) \ll_{d} 1
$$

Remark (1). We deduced the point selection theorem from the multicoloured Tverberg theorem of Živaljević and Vrećica. Now we show, in turn, that the latter follows from the point selection theorem. To see this, take $d+1$ sets $C_{1}, \ldots, C_{d+1}$ in $\mathbb{R}^{d}$, each of cardinality $t$, and set $X=\bigcup_{i=1}^{d+1} C_{i}$. Define $\mathscr{H}$ to be the complete $(d+1)$-partite $(d+1)$-graph with 
$(d+1)$-partition $C_{1}, \ldots, C_{d+1}$. Then $n=t(d+1)$ and $|\mathscr{H}|=t^{d+1} \gg_{d}\left(\begin{array}{c}n \\ d+1\end{array}\right)$. By the point selection theorem, $\mathscr{H}$ has a pierceable subfamily $\mathscr{H}^{\prime}$ of size

$$
\left|\mathscr{H}^{\prime}\right| \gg_{d}\left(\begin{array}{c}
n \\
d+1
\end{array}\right) \gg_{d} t^{d+1}
$$

Consider the largest integer $r$ for which there exist pairwise disjoint $(d+1)$-tuples $S_{1}, \ldots$, $S_{r}$ in $\mathscr{H}^{\prime}$. Then any other $S \in \mathscr{H}^{\prime}$ intersects $\bigcup_{i=1}^{r} S_{i}$, and the number of such $(d+1)$-tuples is at most $(d+1) r t^{d}$. Since here we counted $S_{1}, \ldots, S_{r}$ as well, we get

$$
(d+1) r t^{d} \geq\left|\mathscr{H}^{\prime}\right| \gg_{d} t^{d+1},
$$

which shows that, indeed, $T(r, d) \leq t \ll_{d} r$.

Remark (2). We mention further that the proof method of the point selection theorem cannot give a selection exponent $s_{d}$ smaller than $(d+1)^{(d+1)}$. This is because $T(r, d) \geq r$ implies that $t \geq d+1$ in (6).

\section{Proof of the hitting set theorem}

Proof of Theorem 5.1. We are going to use a greedy algorithm to produce the hitting set E.

Start with $\mathscr{H}=\left(\begin{array}{c}X \\ d+1\end{array}\right)$ and $E=\emptyset$. The algorithm proceeds by choosing a maximal cardinality pierceable subfamily $\mathscr{H}^{\prime} \subset \mathscr{H}$, together with a point $v \in \bigcap$ int $\operatorname{conv}(S): S \in$ $\left.\mathscr{H}^{\prime}\right\}$. Then set $\mathscr{H}=\mathscr{H} \backslash \mathscr{H}^{\prime}$ and $E=E \cup\{v\}$. We stop as soon as $|\mathscr{H}| \leq \eta\left({ }^{n}{ }_{d+1}^{n}\right)$. We claim that when the algorithm stops

$$
|E| \ll_{d} \eta^{1-s}
$$

Assume the algorithm produced the sequence of families $\left(\begin{array}{c}x \\ d+1\end{array}\right)=\mathscr{H}_{0} \supset \mathscr{H}_{1} \supset \ldots \supset \mathscr{H}_{m}$. Denote by $k_{i}$ the index where

$$
\left|\mathscr{H}_{k_{i}}\right| \geq 2^{-i}\left(\begin{array}{c}
n \\
d+1
\end{array}\right) \text { and }\left|\mathscr{H}_{k_{i}+1}\right|<2^{-i}\left(\begin{array}{c}
n \\
d+1
\end{array}\right)
$$

It may happen that $k_{i}=k_{i+1}$, but that will not matter. We know that

$$
\left|\mathscr{H}_{k_{i}+1} \backslash \mathscr{H}_{k_{i+1}+1}\right| \leq\left|\mathscr{H}_{k_{i}+1}\right|<2^{-i}\left(\begin{array}{c}
n \\
d+1
\end{array}\right) .
$$

We also know from the point selection theorem that, for $j \geq k_{i+1}$, we have

$$
\left|\mathscr{H}_{j} \backslash \mathscr{H}_{j+1}\right| \gg_{d}\left(2^{-(i+1)}\right)^{s}\left(\begin{array}{c}
n \\
d+1
\end{array}\right),
$$

since the deleted subfamily $\mathscr{H}_{j} \backslash \mathscr{H}_{j+1}$ was of maximum cardinality. This shows that

$$
k_{i+1}-k_{i} \ll_{d} \frac{2^{-i}\left(\begin{array}{c}
n \\
d+1
\end{array}\right)}{2^{-(i+1) s}\left(\begin{array}{c}
n \\
d+1
\end{array}\right)}=2^{i(s-1)+s} .
$$


Since we stop as soon as $2^{-i} \leq \eta$, i.e., $i \geq\lceil\log 1 / \eta\rceil$, we get that the basic step of the algorithm is carried out

$$
m \leq \sum_{i=0}^{\lceil\log 1 / \eta\rceil}\left(k_{i+1}-k_{i}\right) \ll_{d}^{\lceil} \sum_{i=0}^{\lceil\log 1 / \eta\rceil} 2^{i(s-1)+s} \ll_{d} \eta^{1-s}
$$

times. This proves the claim.

Remark. The hitting set theorem implies the point selection theorem. Indeed, let $\mathscr{H} \subset$ $\left(\begin{array}{c}X \\ d+1\end{array}\right)$ with $|\mathscr{H}|=p\left(\begin{array}{c}n \\ d+1\end{array}\right)$. Set $\eta=p / 2$ and let $E$ be a set of cardinality $O\left(\eta^{-(s-1)}\right)$ missing at most $\eta\left(\begin{array}{c}n \\ d+1\end{array}\right)$ simplices of $X$. Define

$$
\mathscr{H}_{1}=\{S \in \mathscr{H}: E \cap \text { int conv } S \neq \emptyset\} .
$$

Clearly, $\left|\mathscr{H}_{1}\right| \geq \frac{P}{2}\left(\begin{array}{c}n \\ d+1\end{array}\right)$. Since $E$ meets every simplex in $\mathscr{H}_{1}$, there is a point $v \in E$ that is contained in at least

$$
\frac{\left|\mathscr{H}_{1}\right|}{|E|} \gg_{d} p^{s}\left(\begin{array}{c}
n \\
d+1
\end{array}\right)
$$

simplices of $\mathscr{H}_{1}$.

\section{Weak $\varepsilon$-nets for convex sets in $\mathbb{R}^{d}$}

Proof of Theorem 4.1. First we give a simple algorithm producing a weak $\varepsilon$-net $F$, of size $\ll_{d} \varepsilon^{-(d+1)}$.

Start with $F=\emptyset$. Check whether there is a set $Y \subset X,|Y| \geq \varepsilon n$ such that $F$ misses all simplices of $\left(\begin{array}{c}Y \\ d+1\end{array}\right)$. If there is no such $Y$, stop. In this case $F$ is a weak $\varepsilon$-net for $X$. If there is a $Y$ like that, choose a point $v$ common to at least

$$
(1-o(\mathrm{i}))(d+1)^{-d}\left(\begin{array}{c}
|Y| \\
d+1
\end{array}\right)
$$

simplices from $Y$. Such a point exists by (2). Set $F=F \cup\{v\}$.

In each step of the algorithm, the number of missed simplices decreases by at least

$$
(1-o(1))(d+1)^{-d}\left(\begin{array}{c}
|Y| \\
d+1
\end{array}\right) \geq(1-o(1))(d+1)^{-d}\left(\begin{array}{c}
\varepsilon n \\
d+1
\end{array}\right) \gg_{d}\left(\begin{array}{c}
n \\
d+1
\end{array}\right) \varepsilon^{d+1}
$$

Therefore, the algorithm terminates after at most

$$
\frac{\left(\begin{array}{c}
n \\
d+1
\end{array}\right)}{\varepsilon^{d+1}\left(\begin{array}{c}
n \\
d+1
\end{array}\right)} \ll_{d} \varepsilon^{-(d+1)}
$$

steps, showing that $|F| \ll_{d} \varepsilon^{-(d+1)}$.

To get the sharper estimate in the theorem, we apply the previous algorithm, but instead of starting with $F=\emptyset$, we start with $F=E$, where $E$ comes from Theorem 5.1, i.e., $E$ misses at most $\eta\left(\begin{array}{c}n \\ d+1\end{array}\right)$ simplices of $X$ and $|E| \ll_{d} \eta^{1-s}$. 
This time the algorithm terminates after

$$
(1+o(1)) \frac{\eta\left(\begin{array}{c}
n \\
d+1
\end{array}\right)}{(d+1)^{-d}\left(\begin{array}{c}
\varepsilon n \\
d+1
\end{array}\right)} \ll_{d} \eta \varepsilon^{-(d+1)}
$$

steps, producing a weak $\varepsilon$-net $F$ of size $\ll_{d}|E|+\eta \varepsilon^{-(d+1)} \ll_{d} \eta^{1-s}+\eta \varepsilon^{-(d+1)}$. The right choice for $\eta$ is $\varepsilon^{(d+1) / s}$, which gives

$$
|F| \ll_{d} \varepsilon^{-(d+1)+(d+1) / s} .
$$

\section{Weak $\varepsilon$-nets in the plane}

Here we prove Theorem 4.2 by an inductive procedure. Let us start with some remarks and definitions.

Given $k \geq 3$ and a finite set $X \subset \mathbb{R}^{2}$, let $f(X, k)$ denote the minimal size of a weak $\varepsilon$-net for $X$, where $\varepsilon=k /|X|$, i.e.,

$$
f(X, k)=\min \left\{|F|: F \subset \mathbb{R}^{2} \text {, int } \operatorname{conv}(Y) \cap F \neq \emptyset \text { for every } Y \subset X \text { with }|Y| \geq k\right\} .
$$

Note that this definition is stronger than the original, as here we require that $F$ intersect the interior of $\operatorname{conv}(C)$. Consequently, our Theorem 9.1 below is a little stronger than Theorem 4.2. Let $f(n, k)$ be the maximum of $f(X, k)$, where $X$ satisfies (1) with $d=2$, i.e., no three points from $X$ lie on a line. Obviously, we have $f(k, k)=1$, and, more generally, $f(n, k)=1$ if $n \leq k$. In order to bound $f(n, k)$ for small values of $k$, we shall need a result of Katchalski and Meir [13] claiming that

$$
f(n, 3)=2 n-5 \text {. }
$$

Theorem 9.1. $f(n, k)<7(n / k)^{2}$ for all $n \geq k \geq 3$.

Proof. The function $f(n, k)$ is monotonic in the sense that

$$
f(n, k) \geq f\left(n^{\prime}, k^{\prime}\right)
$$

holds for $n \geq n^{\prime}$ and $k \leq k^{\prime}$. Since $7 n^{2} / 25>2 n-5$ for all $n$, relation (8) implies that the theorem holds for $k=3,4$, and 5 . From now on we suppose that $n \geq k \geq 6$.

Now let $X$ be an $n$-set. First, find a line $L$ that bisects $X$ into two parts $X_{1}$ and $X_{2}$ of almost equal size, i.e., $\left|X_{i}\right|=m_{i}$ with $\left|m_{1}-m_{2}\right| \leq 1$. Next we construct a set $V$ such that $V$ intersects int $\operatorname{conv}(Y)$ for every $Y \subset X,|Y| \geq k$ that has more than $\ell$ points on both sides of $L$. (We shall choose $\ell=\lfloor k / 6\rfloor$ later. For the time being we only need $0 \leq \ell \leq(k / 2)-1$.) To this end consider the intersections of $L$ with the line segments connecting $x_{1} \in X_{1}$ to $x_{2} \in X_{2}$. There are $m_{1} m_{2}$ such intersection points, $u(1), \ldots, u\left(m_{1} m_{2}\right)$, indexed consecutively on $L$. (We may suppose that $L$ is in general position with respect to the lines $z_{1} z_{2}$, i.e., all of these intersections are distinct.) Clearly, for any set $Y \subset X$, $|Y| \geq k$ that has at least $\ell+1$ points on both sides of $L, \operatorname{conv}(Y)$ contains at least

$$
h=(\ell+1)(k-\ell-1)
$$


of the $u(i)$ 's. For $V$, choose a point from $L$ between $u(h-1)$ and $u(h), u(2 h-2)$ and $u(2 h-1), u(3 h-3)$ and $u(3 h-2)$, etc. Then

$$
|V|=\left\lceil\frac{m_{1} m_{2}}{h-1}\right\rceil-1 .
$$

What are those sets $Y \subset X,|Y| \geq k$, whose convex hull contains no point from $V$ ? They are the $Y$ that have at most $\ell$ points either in $X_{2}$ or in $X_{1}$. But such a $Y$ must have at least $(k-\ell)$ points in $X_{1}$ (or in $X_{2}$, respectively). So it will be enough to find a weak $\varepsilon_{1}$-net for $X_{1}$, where $\varepsilon_{1}=(k-\ell) / m_{1}$ (and a weak $\varepsilon_{2}$-net for $X_{2}$ with $\left.\varepsilon_{2}=(k-\ell) / m_{2}\right)$. These two sets together with $V$ form a weak $\varepsilon$-net for $X$. Next we apply the induction hypothesis twice, and obtain

$$
f(n, k) \leq f\left(m_{1}, k-\ell\right)+f\left(m_{2}, k-\ell\right)+|V| \leq \frac{7\left(m_{1}^{2}+m_{2}^{2}\right)}{(k-\ell)^{2}}+\frac{m_{1} m_{2}}{(\ell+1)(k-\ell-1)-1} .
$$

Using the facts that $\left(m_{1}^{2}+m_{2}^{2}\right) \leq\left(n^{2}+1\right) / 2, m_{1} m_{2} \leq n^{2} / 4$ and for $k \geq 6, \ell=\lfloor k / 6\rfloor$ one has $(k-\ell)^{2} \geq(25 / 36) k^{2}$ and $(\ell+1)(k-\ell-1)-1 \geq(5 / 36) k^{2}$, we obtain that the right hand side of (9) is at most $(252 / 50)\left(n^{2}+1\right) / k^{2}+(36 / 20)\left(n^{2} / k^{2}\right)$. This is at most $6.98(n / k)^{2}$ for $n \geq k \geq 6$.

Remark. Without finding the fine structure (the clusters) of the set $X$, one cannot obtain a smaller $\varepsilon$-net than $\Omega\left(1 / \varepsilon^{2}\right)$. This can be seen from the following example. Let $C_{1}, C_{2}$, $\ldots, C_{2 / \varepsilon}$ be disjoint, small circular discs in the plane such that there is no point $P$ lying in three of the regions conv $\left(C_{i} \cup C_{j}\right)$, except if all the three contain the same disk $C_{i}$. Put $\varepsilon n / 2$ points around the centre of each $C_{i}$. Then, every $\varepsilon$-net avoiding $\cup C_{i}$ must have at least $\Omega\left(1 / \varepsilon^{2}\right)$ points.

\section{An efficient algorithm to find weak $\varepsilon$-nets}

By applying the results of [15] and [18], one can give an alternative proof of Theorems 4.1 and 4.2 for $d \leq 3$. This proof gives a slightly worse estimate, but has the advantage that it provides an efficient algorithm for constructing the corresponding weak $\varepsilon$-nets. Here is the assertion for the planar case.

Proposition 10.1. For every set $X$ of $n$ points in the plane and for every $\varepsilon>0$, there is a weak $\varepsilon$-net of size $O\left(\varepsilon^{-\log _{4 / 3} 4}\right)$. Such a net can be found in time $O(n \log (1 / \varepsilon))$.

Proof. Without loss of generality, we may assume that $n$ is a power of 4 . By the main result of [15], one can find in time $O(n)$ two intersecting lines $l_{1}$ and $l_{2}$ so that the number of points in each of the four closed regions to which they partition the plane is at least $n / 4$. Let $y$ be the point of intersection of these two lines, and partition $X$ into four pairwise disjoint subsets $X_{1}, \ldots, X_{4}$ of cardinality $n / 4$ each, where each $X_{i}$ is completely contained in one of the above closed regions. Observe that if a convex set contains at least one point from each $X_{i}$, then it contains $y$, i.e., $Y=\{y\}$ is a weak $3 / 4$-net for $X$.

It follows that any convex set that does not contain $y$ misses completely at least one of the sets $X_{i}$, and hence, if it contains at least $\varepsilon n$ points of $X$, then it contains at least 
a fraction $(4 / 3) \varepsilon$ of one of the sets $X_{i}$. Therefore, by recursively constructing $(4 / 3) \varepsilon$-nets in each $X_{i}$ we conclude that the size $f(\varepsilon)$ of our net satisfies $f(\varepsilon) \leq 1+4 f\left(\frac{4}{3} \varepsilon\right)$ (and $f(\delta)=1$ for all $\delta \geq 3 / 4)$. This easily gives the bound stated above. The time $t(n, \varepsilon)$ for finding the net in our construction satisfies $t(n, \varepsilon) \leq O(n)+4 t\left(n / 4, \frac{4}{3} \varepsilon\right)$, which implies that $t(n, \varepsilon) \leq O(n \log (1 / \varepsilon))$, completing the proof.

\section{References}

[1] Alon, N. and Kleitman, D. J. (to appear) Piercing convex sets and the Hadwiger-Debrunner (p,q)-problem. Adv. Math.

[2] Aronov, B., Chazelle, B., Edelsbrunner, H., Guibas, L. J., Sharir, M. and Wenger, R. (1991) Points and triangles in the plane and halving planes in space. Discrete Comp. Geom. 6 435-442.

[3] Bárány, I. (1982) A generalization of Caratheodory's theorem. Discrete Math. 40 141-152.

[4] Bárány, I., Füredi, Z. and Lovász, L. (1990) On the number of halving planes. Combinatorica 10 175-183.

[5] Bárány, I. and Larman, G. D. (to appear) A coloured version of Tverberg's theorem. J. London Math. Soc.

[6] Beck, J. and Chen, W. (1987) Irregularities of distributions. Cambridge Tracts in Math. 89, Cambridge University Press.

[7] Boros, E. and Füredi, Z. (1984) The number of triangles covering the center of an $n$-set. Geom. Dedicata 17 69-77.

[8] Capoyleas, V. (1991) An upper bound for weak ह-nets of points in convex position: preliminary version, (manuscript).

[9] Chernoff, H. (1952) A measure of asymptotic efficiency for tests of an hypothesis based on the sum of observations. Ann. Math. Statist. 23 497-507.

[10] Erdős, P. (1964) On extremal problems of graphs and generalized graphs. Israel J. Math. 2 183-190.

[11] Erdős, P. and Simonovits, M. (1983) Supersaturated graphs and hypergraphs. Combinatorica 3 181-192.

[12] Jaromczyk, J. W. and Światek, G. (1990) The optimal constant for the colored version of Tverberg's theorem. DIMACS Technical Report 90-6.

[13] Katchalski, M. and Meir, A. (1988) On empty triangles determined by points in the plane. Acta Math Hungar. 51 323-328.

[14] Lovász, L. (1971) On the number of halving lines. Annales Univ Sci Budapest, Eötvös 14 $107-108$.

[15] Megiddo, N. (1985) Partitioning with two lines in the plane. J. Algorithms 6 430-433.

[16] Tverberg, H. (1966) A generalization of Radon's theorem. J. London Math. Soc. 41 123-128.

[17] Welzl, E. (1990) Lecture at a DIMACS conference, January 1990.

[18] Yao, F. F. (1983) A 3-space partition and its applications. Proc. 15th Annual ACM STOC, ACM Press, New York, 258-263.

[19] Živaljević, R. T. and Vrećica, S. T. (to appear) The coloured Tverberg problem and complexes of injective functions. J. Comb. Theory, $A$ 